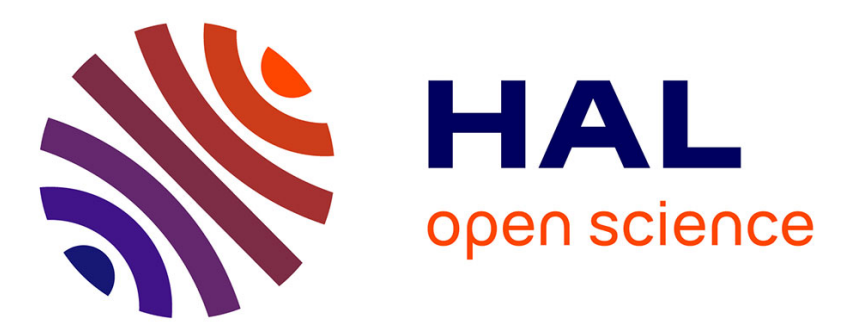

\title{
Optimal Control of an Ensemble of Bloch Equations with Applications in MRI
}

Bernard Bonnard, Alain Jacquemard, Jérémy Rouot

\section{To cite this version:}

Bernard Bonnard, Alain Jacquemard, Jérémy Rouot. Optimal Control of an Ensemble of Bloch Equations with Applications in MRI. 55th IEEE Conference on Decision and Control - CDC, Dec 2016, Las Vegas, United States. pp.1608-1613, 10.1109/CDC.2016.7798495 . hal-01287290v4

\section{HAL Id: hal-01287290 \\ https://hal.inria.fr/hal-01287290v4}

Submitted on 8 Dec 2016

HAL is a multi-disciplinary open access archive for the deposit and dissemination of scientific research documents, whether they are published or not. The documents may come from teaching and research institutions in France or abroad, or from public or private research centers.
L'archive ouverte pluridisciplinaire $\mathbf{H A L}$, est destinée au dépôt et à la diffusion de documents scientifiques de niveau recherche, publiés ou non, émanant des établissements d'enseignement et de recherche français ou étrangers, des laboratoires publics ou privés. 


\title{
Optimal Control of an Ensemble of Bloch Equations with Applications in MRI
}

\author{
Bernard Bonnard and Alain Jacquemard and Jérémy Rouot
}

\begin{abstract}
The optimal control of an ensemble of Bloch equations describing the evolution of an ensemble of spins is the mathematical model used in Nuclear Resonance Imaging and the associated costs lead to consider Mayer optimal control problems. The Maximum Principle allows to parameterize the optimal control and the dynamics is analyzed in the framework of geometric optimal control. This leads to numerical implementations or suboptimal controls using averaging principle.
\end{abstract}

\section{INTRODUCTION}

Optimal control algorithm was introduced in [1] in Nuclear Magnetic Resonance to improve the control field and at the end of the nineties, new theoretical results appear in optimal control of NMR systems both from the analytical and numerical points of view [8],[13],[10]. More recently under the impulse of S.J. Glaser, combination of geometric control based on the Maximum Principle [12] and related numerical algorithms ((Gradient methods: Grape) or shooting and continuation methods (HamPath [6])) were used and lead to sophisticated results starting from a complete solution of the time minimal saturation of a single spin [9] and application to the contrast problem in Magnetic Resonance Imaging, see [4],[11].

The model for analyzing such problems is to consider an ensemble of $\mathrm{N}$-spins, each spin being described by a magnetization vector: $M_{i}=\left(M_{x_{i}}, M_{y_{i}}, M_{z_{i}}\right)$ in a (fixed) laboratory frame and satisfying the so-called Bloch equation:

$$
\frac{\mathrm{d} M_{i}}{\mathrm{~d} t}=\gamma M_{i} \wedge B+R(M),
$$

$\gamma$ is the gyromagnetic ratio, $B(t) \in \mathbb{R}^{3}$ is the total magnetic field applied to the system which decomposes into:

$$
B(t)=B_{0}(t)+B_{1}(t)
$$

where $B_{0}(t)$ is oriented along the $z$-axis while $B_{1}(t)$ is the control RF-field in the transverse plane $(x, y)$. In Magnetic Resonance $B_{0}(t)$ decomposes into $B_{0}+\Delta B_{0}(X, Y, t)$ where $B_{0}$ is an intense stationary field. In this article we shall restrict to the case $\Delta B_{0}(X, Y)$ associated with MRI, where $X, Y$ describe the spatial position of the voxel in the image, time dependance has to be taken into account in Magnetic

B. Bonnard and A. Jacquemard are with Institut de Mathématiques de Bourgogne, Université de Bourgogne, 9 avenue Alain Savary, 21078 Dijon, France, bernard.bonnard@u-bourgogne.fr, alain.jacquemard@ubourgogne.fr

B. Bonnard and J. Rouot are with Inria, Sophia Antipolis - Mediterranée Center, 06902 Sophia Antipolis, France, jeremy.rouot@inria.fr

A. Jacquemard is with Sorbonne Universités, UPMC Univ Paris 06, CNRS, INRIA, Laboratoire d'Informatique de Paris 6 (LIP6), Équipe PolSys, 4 place Jussieu, 75252 Paris Cedex 05, France
Resonance Elastography where our computations can be easily generalized. The term $R(M)$ is the dissipation of the form:

$$
R(M)=\left(M_{x_{i}} / T_{2}^{i}, M_{y_{i}} / T_{2}^{i},\left(M_{z_{i}}-M_{0 i}\right) / T_{1}^{i}\right)
$$

where $M_{0 i}$ is the equilibrium magnetization which can be normalized to 1 , using rescaling $M_{i} \rightarrow M_{i} / M_{0 i}$ and $T_{1}^{i}, T_{2}^{i}$ are the relaxation parameters which are the chemical signatures of the observed species. The control components are denoted $u(t)=-\gamma B_{y}(t), \quad v(t)=-\gamma B_{x}(t)$ where $B_{1}=\left(B_{x}, B_{y}\right)$ and up to a time rescaling one can impose the control bound: $u^{2}+v^{2} \leq 1$.

An important step is to rewrite each Bloch equation in a rotating frame:

$$
M_{i}(t)=S(t) q_{i}(t), q_{i}=\left(x_{i}, y_{i}, z_{i}\right)
$$

with

$$
S(t)=\exp \left(\omega t \Omega_{z}\right), \Omega_{z}=\left(\begin{array}{ccc}
0 & -1 & 0 \\
1 & 0 & 0 \\
0 & 0 & 0
\end{array}\right)
$$

and the Bloch equation takes the form:

$$
\frac{\mathrm{d}}{\mathrm{d} t}\left(\begin{array}{c}
x_{i} \\
y_{i} \\
z_{i}
\end{array}\right)=\left(\begin{array}{ccc}
-1 / T_{2}^{i} & -\Delta \omega_{i} & u_{2} \\
\Delta \omega_{i} & -1 / T_{2}^{i} & -u_{1} \\
-u_{2} & u_{1} & -1 / T_{1}^{i}
\end{array}\right)\left(\begin{array}{c}
x_{i} \\
y_{i} \\
z_{i}
\end{array}\right)+\left(\begin{array}{c}
0 \\
0 \\
1 / T_{1}^{i}
\end{array}\right)
$$

where $\Delta \omega_{i}$ is the resonance offset and the RF-control field is represented using the rotation:

$$
u_{2}=u \cos (\omega t)-v \sin (\omega t), u_{1}=u \sin (\omega t)+v \cos (\omega t)
$$

which preserves the control bound: $u_{1}^{2}+u_{2}^{2} \leq 1$.

Finally in the moving frame, the Bloch equation takes the normalized form uses in our computations:

$$
\begin{aligned}
\frac{\mathrm{d} x_{i}}{\mathrm{~d} t} & =-\Gamma_{i} x_{i}-\Delta \omega_{i} y_{i}+u_{2} z_{i} \\
\frac{\mathrm{d} y_{i}}{\mathrm{~d} t} & =\Delta \omega_{i} x_{i}-\Gamma_{i} y_{i}-u_{1} z_{i} \\
\frac{\mathrm{d} z_{i}}{\mathrm{~d} t} & =\gamma_{i}\left(1-z_{i}\right)+u_{1} y_{i}-u_{2} x_{i}
\end{aligned}
$$

where $2 \Gamma_{i} \geq \gamma_{i} \geq 0$ so that the Bloch ball $\left|q_{i}\right| \leq 1$ is invariant.

The case $\Delta \omega_{i}=0$ is called the resonant case.

Collecting $\mathrm{N}$-spins systems, each state being given by $q_{i}=$ $\left(x_{i}, y_{i}, z_{i}\right), i=1, \ldots, N$ and denoting $q=\left(q_{1}, \ldots, q_{N}\right)$ the state describing the ensemble of $\mathrm{N}$-spins, the system takes the form:

$$
\frac{\mathrm{d} q}{\mathrm{~d} t}=F_{0}(q)+u_{1} F_{1}(q)+u_{2} F_{2}(q)
$$


where thanks to the Bloch equation, $F_{0}, F_{1}, F_{2}$ are a copy of $\mathrm{N}$-affine vectors fields, each depending linearly on the dissipation parameters $\Gamma_{i}, \gamma_{i}$ and of the resonance offset $\Delta \omega_{i}$.

The optimal control problems related to our applications consists into a Mayer problem, optimizing a cost function

$$
c\left(q\left(t_{f}\right)\right) \rightarrow \operatorname{Min},
$$

with initial condition $q(0)$ and a terminal condition defined by $f\left(q\left(t_{f}\right)\right)=0$ where $f: \mathbb{R}^{3 N} \rightarrow \mathbb{R}^{k}$ and $t_{f}$ is the fixed transfer time.

The contribution of this article is to analyze the above mentioned problems, using the Maximum Principle and to derive a computation of the optimal control taking into account the specificity of the problem, that is: the control is bi-input and the control system is described by a set of $\mathrm{N}$-Bloch equations. In particular, the contrast problem in MRI will be studied in details to derive the properties in the resonant case for $N=2$. The general case taking into account the $B_{0}$ and $B_{1}$-inhomogeneities can be understood in this framework and suboptimal control can be derived using appropriate averaging procedure or numerically computed. The principle is coming from our model. Indeed if $N=2$, the optimal control can be computed in the resonant frame to derive the physical control $u(t), v(t)$. But coupling such systems leads to compute the optimal solution as an averaged process given by the Maximum Principle of the individual controls of each problems of the form $u(t)=-u_{1} \sin \left(\omega_{i} t\right)+$ $u_{2} \cos \left(\omega_{i} t\right), v(t)=u_{1} \cos \left(\omega_{i} t\right)+u_{2} \sin \left(\omega_{i} t\right)$ where $\omega_{i}$ is the resonant frequency and $u_{1}, u_{2}$ are the optimal controls. This corresponds to the concept of choregraphy (see [3] for related numerical simulations).

\section{MAXimum PRinciple AND Optimal CONTROL COMPUTATIONS}

\section{A. Preliminaries}

We consider the Mayer problem associated with the control system (real analytic data): $\frac{\mathrm{d} q}{\mathrm{~d} t}=F(q, u), q \in$ $Q \subset \mathbb{R}^{n}$ with fixed initial condition $q(0)=q_{0}$, where the set of admissible controls $\mathcal{U}$ is the set $u \in L^{\infty}\left(\left[0, t_{f}\right]\right)$, valued in the control domain $U$, such that the associated trajectory $q\left(t, q_{0}, u\right), q(0)=q_{0}$ is defined on $\left[0, t_{f}\right]$ and let $A\left(q_{0}, t_{f}\right)=\cup_{u \in \mathcal{U}} q\left(t_{f}, q_{0}, u\right)$ be the accessibility set, at time $t_{f}$. The cost function is denoted $c$, and the problem is of the form: $\operatorname{Min}_{u \in \mathcal{U}} c\left(t_{f}\right)$ and the boundary conditions are defined by $f\left(q\left(t_{f}\right)\right)=0, f: \mathbb{R}^{n} \rightarrow \mathbb{R}^{k}$. For fixed $m$, we define $M_{m}=\{q ; f(q)=0, c(q)=m\}$, where $m$ is the minimal cost. The geometric interpretation of the Maximum Principle leads to define the adjoint vector as orthogonal to the hyperplane separating $A\left(q_{0}, T\right)$ from $M_{m}$, this giving the following necessary conditions.

Proposition 1: Assume $u^{\star}$ with corresponding trajectory $q^{\star}$ optimal on $\left[0, t_{f}\right]$, then the following necessary conditions are satisfied. Introducing $H(z, u)=\langle p, F(q, u)\rangle$ with $z=$ $(q, p)$ called the pseudo-Hamiltonian, then there exists $p^{\star}($. such that for almost every $t \in\left[0, t_{f}\right]$ : (i) $\frac{\mathrm{d} q^{\star}}{\mathrm{d} t}=\frac{\partial H}{\partial p}\left(z^{\star}, u^{\star}\right), \frac{\mathrm{d} p^{\star}}{\mathrm{d} t}=-\frac{\partial H}{\partial q}\left(z^{\star}, u^{\star}\right)$,

(ii) $H\left(z^{\star}, u^{\star}\right)=\operatorname{Max}_{u \in U} H\left(z^{\star}, u\right)$ (maximization condition) and the following boundary conditions:

(iii) $f\left(q^{\star}\left(t_{f}\right)\right)=0$,

(iv) $p^{\star}\left(t_{f}\right)=p_{0} \frac{\partial c}{\partial q}\left(q^{\star}\left(t_{f}\right)\right)+\sum_{i=1}^{k} \sigma_{i} \frac{\partial f_{i}}{\partial q}\left(q^{\star}\left(t_{f}\right)\right)=$ $0, p_{0} \leq 0$ (transversality condition).

Definition 1: A solution of conditions (i) and (ii) of the Maximum Principle is called an extremal and if it satisfies the boundary conditions, it is called a BC-extremal.

1) Application to MRI: One considers an ensemble of Nspins associated with the contrast problem, with saturation. The necessary optimal conditions are easily deduced.

a) Ideal contrast: (without $B_{0}, B_{1}$ inhomogeneities). One takes $N=2, q_{1}$ : magnetization vector species $1, q_{2}$ : magnetization vector species 2. Saturation of the first spin gives: $q_{1}\left(t_{f}\right)=0$ and maximization of the contrast of the second spin writes: $\operatorname{Max}\left|q_{2}\left(t_{f}\right)\right|$ and the cost function is: $c(q)=\left|q_{2}\right|^{2}$. Splitting $p$ into $\left(p_{1}, p_{2}\right)$, the transversality condition is: $p_{2}\left(t_{f}\right)=-2 p_{0} q_{2}\left(t_{f}\right), p_{0} \leq 0$. If $p_{0} \neq 0$, it can be normalized to $p_{0}=-1 / 2$.

b) Contrast with $B_{0}$-inhomogeneities: We consider an ensemble of $N$-voxels. The state is $q=\left(q_{1}^{i}, q_{2}^{i}\right), i=$ $1, \ldots, N$ where $q_{1}, q_{2}$ are the two chemical species. Each voxel is associated with a pair of Bloch equation (4) with parameter $\left(\Gamma_{1}, \gamma_{1}\right),\left(\Gamma_{2}, \gamma_{2}\right)$ and resonance offset $\Delta \omega_{i}$. Exact saturation leads to: $q_{1}^{i}\left(t_{f}\right)=0, i=1, \ldots, N$ and the cost takes the form $\sum_{i=1}^{N} q_{2}^{i^{2}}\left(t_{f}\right)$. Another point of view is to relax the boundary condition and to consider a cost:

$$
c_{\alpha}(q)=\alpha\left(\sum_{i=1}^{N} q_{1}^{i^{2}}\left(t_{f}\right)\right)-\sum_{i=1}^{N}{q_{2}^{i}}^{2}\left(t_{f}\right)
$$

where $\alpha$ is a parameter.

c) Contrast with $B_{1}$-inhomogeneities: The effect of $B_{1}$-inhomogeneities on a given voxel is to make a variation of the maximal control amplitude $u_{1}^{2}+u_{2}^{2}$, which has been normalized to 1 . Hence one must replace the control vector fields $F_{j}, j=1,2$ by $\left(1-\varepsilon_{i}\right) F_{j}$ where $\varepsilon_{i}$ corresponds to voxel $i$.

\section{B. Lie brackets computations}

The importance feature of the system is the possibility of performing all Lie brackets computations and relations between Lie brackets using the invariance of the system on an appropriate semi-direct Lie group, we refer to [7] for the theoretical framework.

1) Preliminaries: First of all we use the following Lie and Poisson brackets relations. If $X, Y$ are two (smooth) vector fields on $Q$, the Lie bracket is defined by:

$$
[X, Y](q)=\frac{\partial X}{\partial q} Y(q)-\frac{\partial Y}{\partial q} X(q) .
$$

If $H$ is an Hamiltonian on $T^{\star} Q$, one denotes $\vec{H}(z)=$ $\left(\frac{\partial H}{\partial p},-\frac{\partial H}{\partial q}\right)$ and the Poisson bracket of $H_{1}, H_{2}$ is defined by:

$$
\left\{H_{1}, H_{2}\right\}(z)=\mathrm{d} H_{1}\left(\overrightarrow{H_{2}}(z)\right)
$$


Let $X_{1}, X_{2}$ be two vector fields on $Q$ defining the two Hamiltonians $H_{i}(z)=\left\langle p, F_{i}(q)\right\rangle, i=1,2$. One has:

$$
\left\{H_{1}, H_{2}\right\}(z)=\left\langle p,\left[F_{1}, F_{2}\right](q)\right\rangle .
$$

For a single voxel, one can assume that $\Delta \omega_{i}=0$, and omitting the indices the Bloch equation (4) can be written:

$$
\frac{\mathrm{d} q}{\mathrm{~d} t}=F_{0}(q)+u_{1} F_{1}(q)+u_{2} F_{2}(q) .
$$

Next we present all Lie brackets computations and relations crucial in our analysis.

2) Lie brackets of length less than 4: The indices are omitted. To take into account the detuning for an ensemble of spins we introduce:

$$
\widetilde{F_{0}}=(-\Gamma x-\varepsilon y,-\Gamma y+\varepsilon, \gamma(1-z))=F_{0}+\varepsilon(-y, x, 0)
$$

where $(-y, x, 0)$ corresponds to a rotation along the $\mathrm{z}$-axis. Note that $\varepsilon$ can be taken as zero for a single voxel using a resonant representation. We write $F_{1}=(0,-z, y), F_{2}=$ $(z, 0,-x)$ and we have the following Lie brackets

- Length 2:

$$
\begin{aligned}
& {\left[\widetilde{F_{0}}, F_{1}\right]=\left[F_{0}, F_{1}\right]+\varepsilon F_{2}=(z \varepsilon, \gamma-\delta z,-\delta y-\varepsilon x)} \\
& {\left[\widetilde{F_{0}}, F_{2}\right]=\left[F_{0}, F_{1}\right]-\varepsilon F_{1}=(-\gamma+\delta z, z \varepsilon, \delta x-\varepsilon y)} \\
& {\left[F_{1}, F_{2}\right]=(-y, x, 0)}
\end{aligned}
$$

- Length 3:

$$
\begin{aligned}
{\left[\left[F_{1}, F_{2}\right], F_{1}\right] } & =F_{2},\left[\left[F_{1}, F_{2}\right], \widetilde{F_{0}}\right]=0,\left[\left[F_{1}, F_{2}\right], F_{2}\right]=-F_{1} \\
{\left[\left[\widetilde{F}_{0}, F_{1}\right], F_{1}\right] } & =\left[\left[F_{0}, F_{1}\right], F_{1}\right]-\varepsilon\left[F_{1}, F_{2}\right]=(\varepsilon y,-2 \delta y-\varepsilon x,-\gamma+2 \delta z) \\
{\left[\left[\widetilde{F}_{0}, F_{1}\right], F_{2}\right] } & =(\delta y, \delta x, 0)=\left[\left[\widetilde{F}_{0}, F_{2}\right], F_{1}\right] \\
{\left[\left[\widetilde{F}_{0}, F_{2}\right], F_{2}\right] } & =\left[\left[F_{0}, F_{2}\right], F_{2}\right]-\varepsilon\left[F_{1}, F_{2}\right]=(-2 \delta x+\varepsilon y,-\varepsilon x, 2 \delta z-\gamma) \\
{\left[\left[\widetilde{F}_{0}, F_{1}\right], \widetilde{F_{0}}\right] } & =\left[\left[F_{0}, F_{1}\right], F_{0}\right]+\varepsilon\left(\left[\left[F_{0}, F_{1}\right],\left[F_{1}, F_{2}\right]\right]+\left[F_{2}, F_{0}\right]\right)+\varepsilon^{2} F_{1} \\
& =\left(-2(\delta z-\gamma) \varepsilon,-\gamma(\gamma-2 \Gamma)+\delta^{2} z-z \varepsilon^{2},-\delta^{2} y-2 \varepsilon x \delta+\varepsilon^{2} y\right) \\
{\left.\left[\widetilde{F_{0}}, F_{2}\right], \widetilde{F_{0}}\right] } & =\left[\left[F_{0}, F_{2}\right], F_{0}\right]+\varepsilon\left(\left[\left[F_{0}, F_{2}\right],\left[F_{1}, F_{2}\right]\right]-\left[F_{1}, F_{0}\right]\right)+\varepsilon^{2} F_{2} \\
& =\left(\gamma(\gamma-2 \Gamma)-\delta^{2} z+\varepsilon^{2} z,-2(\delta z-\gamma) \varepsilon, \delta^{2} x-2 \varepsilon y \delta-\varepsilon^{2} x\right)
\end{aligned}
$$

C. Application to the stratified computations of the extremal solutions

First of all, one needs to introduce the concept of singular extremals, adapted to our study, see [2] for more details.

1) Singular extremals: Consider the (smooth) control system: $\dot{q}=F(q, u), q \in \mathbb{R}^{n}$, and the set of admissible controls is the set $\mathcal{U}$ of bounded measurable mappings $u$ defined on $\left[0, t_{f}\right]$ and valued in an open set $U$. Denoting $q\left(t, x_{0}, u\right)$ the solution emanating from $q_{0}$, the input state mapping (fixed $\left.q_{0}, t_{f}\right)$ is the mapping $E: u \in \mathcal{U} \mapsto q\left(t_{f}, q_{0}, u\right)$. The set of admissible controls is endowed with the $L^{\infty}$-norm: $\|u\|=\sup _{t \in\left[0, t_{f}\right]}|u(t)|$.

Definition 2: A control $u$ is singular on $\left[0, t_{f}\right]$ if the Fréchet derivative of $E$, denoted $E^{\prime}$ is not of full rank when evaluated at $u$ and the corresponding trajectory $q\left(., q_{0}, u\right)$ is called singular on $\left[0, t_{f}\right]$.

One has:

Proposition 2: If $u($.$) is singular on \left[0, t_{f}\right]$ there exists $p($.$) on \left[0, t_{f}\right]$ such that $(q, p, u)$ is solution of the equations:

$$
\dot{q}=\frac{\partial H}{\partial p}, \dot{p}=-\frac{\partial H}{\partial p}, \frac{\partial H}{\partial u}=0
$$

with $H(z, u)=\langle p, F(q, u)\rangle$ is the pseudo-Hamiltonian.

The corresponding triplet $(z(),. u()$.$) is called a singular$ extremal on $\left[0, t_{f}\right]$ for the corresponding control domain $U$.

2) Extremals of order zero: Denoting $H_{i}(z)=$ $\left\langle p, F_{i}(q)\right\rangle, i=0,1,2$ the Hamiltonian lifts and the maximization condition of the Maximum Principle leads to:

$$
u_{1}=\frac{H_{1}}{\sqrt{H_{1}^{2}+H_{2}^{2}}}, \quad u_{2}=\frac{H_{2}}{\sqrt{H_{1}^{2}+H_{2}^{2}}}
$$

if $z$ doesn't belong to the (switching) surface $\Sigma: H_{1}=$ $H_{2}=0$. The corresponding extremals are called of order zero and they are the (smooth) solutions of the Hamiltonian $H_{n}=H_{0}+\left(H_{1}^{2}+H_{2}^{2}\right)^{1 / 2}$

They have the following interpretation.

Proposition 3: Extremals of order zero corresponds to singularities of the input-state mapping, restricting $u$ to a chart of $S^{1}: u_{1}^{2}+u_{2}^{2}=1$.

Additional extremals are contained in the surface $\Sigma$ and we have the following.

Proposition 4: Extremals contained in $\Sigma$ correspond to singularities of the input state mapping, corresponding to the bi-input control systems.

Next they are computed in the case $N=2$ and restricting to the resonant situation. The generalization being straightforward. In our computations, we take into account the control constraints: $u_{1}^{2}+u_{2}^{2} \leq 1$ and the so-called Goh condition $\left\{H_{1}, H_{2}\right\}=0$ related to optimality issues, see [2].

a) Stratification of the surface $\Sigma: H_{1}=H_{2}=0$ : Let $z$ be a solution of $\overrightarrow{H_{0}}+u_{1} \overrightarrow{H_{1}}+u_{2} \overrightarrow{H_{2}}$. Differentiating $H_{1}$ and $H_{2}$ along such a solution one gets:

$$
\begin{gathered}
\dot{H}_{1}=\left\{H_{1}, H_{0}\right\}+u_{2}\left\{H_{1}, H_{2}\right\} \\
\dot{H}_{2}=\left\{H_{2}, H_{0}\right\}-u_{1}\left\{H_{1}, H_{2}\right\} .
\end{gathered}
$$

Hence we have:

Proposition 5: Let $z_{0} \in \Sigma_{1}=\Sigma \backslash\left\{H_{1}, H_{2}\right\}=0$, and define the control $u_{s}^{1}$ by

$$
u_{s}^{1}(z)=\frac{\left(\left\{H_{2}, H_{0}\right\}(z),\left\{H_{0}, H_{1}\right\}(z)\right)}{\left\{H_{1}, H_{2}\right\}(z)}
$$

and plugging $u_{s}^{1}(z)$ into $H$ defined the true Hamiltonian, whose solutions parameterized the singular extremal contained in $\Sigma_{1}$.

One can easily proves that the corresponding control is such that $\left|u_{s}^{1}\right|>1$ and moreover the Goh condition is not satisfied. Hence, they play no role in our analysis.

Next, we consider extremals in $\Sigma$ such that the Goh relation is satisfied that is:

$$
H_{1}=H_{2}=\left\{H_{1}, H_{2}\right\}=0
$$

Using (5) one gets the additional conditions by differentiating:

$$
\left\{H_{1}, H_{2}\right\}=\left\{H_{0}, H_{1}\right\}=\left\{H_{0}, H_{2}\right\}=0
$$

Then differentiating again one gets:

$$
\begin{aligned}
\left\{\left\{H_{1}, H_{2}\right\}, H_{0}\right\}+u_{1}\{ & \left.\left\{H_{1}, H_{2}\right\}, H_{1}\right\} \\
& +u_{2}\left\{\left\{H_{1}, H_{2}\right\}, H_{2}\right\}=0
\end{aligned}
$$




$$
\begin{aligned}
\left\{\left\{H_{0}, H_{1}\right\}, H_{0}\right\}+u_{1}\{ & \left.\left\{H_{0}, H_{1}\right\}, H_{1}\right\} \\
& +u_{2}\left\{\left\{H_{0}, H_{1}\right\}, H_{2}\right\}=0 \\
\left\{\left\{H_{0}, H_{2}\right\}, H_{0}\right\}+u_{1}\{ & \left.\left\{H_{0}, H_{2}\right\}, H_{1}\right\} \\
& +u_{2}\left\{\left\{H_{0}, H_{2}\right\}, H_{2}\right\}=0
\end{aligned}
$$

This leads in general to three relations to compute two control components and according to Lie brackets computations, we have:

Lemma 1: If $H_{1}=H_{2}=0$, one has

$$
\begin{aligned}
& \left\{\left\{H_{1}, H_{2}\right\}, H_{0}\right\}=0,\left\{\left\{H_{1}, H_{2}\right\}, H_{1}\right\}=H_{2}=0, \\
& \left\{\left\{H_{1}, H_{2}\right\}, H_{2}\right\}=-H_{1}=0 . \triangleleft
\end{aligned}
$$

The equation (9) are then written: $\tilde{A}+\tilde{B} u$ and if $\operatorname{det}(\tilde{B}) \neq$ 0 , the corresponding singular control is given by:

$$
u_{s}^{2}(z)=-\tilde{B}^{-1}(z) \tilde{A}(z)
$$

Using the relations:

$$
H_{1}=H_{2}=\left\{H_{1}, H_{2}\right\}=\left\{H_{0}, H_{1}\right\}=\left\{H_{0}, H_{2}\right\}=0,
$$

the vector $p$ is orthogonal to $F_{1}, F_{2},\left[F_{1}, F_{2}\right],\left[F_{0}, F_{1}\right]$, $\left[F_{0}, F_{2}\right]$. Introducing:

$$
\begin{aligned}
& A=\left(\begin{array}{l}
A_{1} \\
A_{2}
\end{array}\right), B=\left(\begin{array}{ll}
B_{1} & B_{3} \\
B_{2} & B_{4}
\end{array}\right), \\
& C=\left(F_{1}, F_{2},\left[F_{1}, F_{2}\right],\left[F_{0}, F_{1}\right],\left[F_{0}, F_{2}\right]\right),
\end{aligned}
$$

with

$$
A_{1}=\operatorname{det}\left(C,\left[\left[F_{0}, F_{1}\right], F_{0}\right]\right), \quad A_{2}=\operatorname{det}\left(C,\left[\left[F_{0}, F_{2}\right], F_{0}\right]\right),
$$

and

$$
\begin{array}{ll}
B_{1}=\operatorname{det}\left(C,\left[\left[F_{0}, F_{1}\right], F_{1}\right]\right), & B_{2}=\operatorname{det}\left(C,\left[\left[F_{0}, F_{2}\right], F_{1}\right]\right), \\
B_{3}=\operatorname{det}\left(C,\left[\left[F_{0}, F_{1}\right], F_{2}\right]\right), & B_{4}=\operatorname{det}\left(C,\left[\left[F_{0}, F_{2}\right], F_{2}\right]\right),
\end{array}
$$

the relation (9) leads to:

$$
A+B u=0
$$

and if $\operatorname{det} B \neq 0$, one gets the singular control given by the feedback:

$$
u_{s}^{2}(q)=-B^{-1}(q) A(q)
$$

and the associated vector field:

$$
Q_{s}^{2}=F_{0}+u_{s, 1}^{2} F_{1}+u_{s, 2}^{2} F_{2}
$$

Moreover, the singular control has to be admissible: $\left|u_{s}^{2}\right| \leq 1$. We introduce the stratum:

$\Sigma_{2}: H_{1}=H_{2}=\left\{H_{1}, H_{2}\right\}=\left\{H_{0}, H_{1}\right\}=\left\{H_{0}, H_{2}\right\} \backslash \operatorname{det} \tilde{B}=0$.

Hence we have:

Lemma 2: 1) On the stratum $\Sigma_{2}$, there exist singular extremals satisfying Goh condition where the singular control is given by the feedback (10).
2) For the contrast problem:

$$
\begin{aligned}
\operatorname{det} B & =-\left(y_{2} x_{1}-x_{2} y_{1}\right)^{4}\left(\Gamma_{1}-\Gamma_{2}-\gamma_{1}+\gamma_{2}\right) \\
& \left(2 \Gamma_{1}{ }^{2} \gamma_{2} z_{1}-2 \Gamma_{1} \Gamma_{2} \gamma_{1} z_{2}+2 \Gamma_{1} \Gamma_{2} \gamma_{2} z_{1}\right. \\
& -4 \Gamma_{1} \gamma_{1} \gamma_{2} z_{1}+2 \Gamma_{1} \gamma_{1} \gamma_{2} z_{2}-2 \Gamma_{1} \gamma_{2}{ }^{2} z_{1} \\
& -2 \Gamma_{2}{ }^{2} \gamma_{1} z_{2}+2 \Gamma_{2} \gamma_{1}^{2} z_{2}-2 \Gamma_{2} \gamma_{1} \gamma_{2} z_{1} \\
& +4 \Gamma_{2} \gamma_{1} \gamma_{2} z_{2}+2 \gamma_{1}{ }^{2} \gamma_{2} z_{1}-2 \gamma_{1}{ }^{2} \gamma_{2} z_{2} \\
& +2 \gamma_{1} \gamma_{2}^{2} z_{1}-2 \gamma_{1} \gamma_{2}^{2} z_{2}+\Gamma_{1} \gamma_{1} \gamma_{2}-\Gamma_{2} \gamma_{1} \gamma_{2} \\
& \left.-\gamma_{2} \gamma_{1}^{2}+\gamma_{1} \gamma_{2}^{2}\right) . \triangleleft
\end{aligned}
$$

Finally, another important property of the extremal flow which is a consequence of the symmetry of revolution, is given next, in relation with Goh condition. It is an application of Noether integrability theorem.

Proposition 6: In the contrast problem, for the Hamiltonian vector field $\vec{H}_{n}$ whose solutions are extremals of order zero, the Hamiltonian lift $H(z)=\left\{H_{1}, H_{2}\right\}(z)=$ $\left(p_{y_{1}} x_{1}-p_{x_{1}} y_{1}\right)+\left(p_{y_{2}} x_{2}-p_{x_{2}} y_{2}\right)$ is a first integral.

The case $\boldsymbol{u}_{2}=\mathbf{0}$ : Another consequence of the symmetry of revolution and enlighten by the previous computation is the following. There exist singular extremals such that $\operatorname{det} B=0$ since the plane $x_{1}=x_{2}=0$ can be made invariant imposing $u_{2}=0$. The system behaves as the single-input system $\frac{\mathrm{d} q}{\mathrm{~d} t}=F_{0}(q)+u_{1} F_{1}(q)$, thus defining the singular control:

$$
u_{1, s}=-\frac{\left\{\left\{H_{0}, H_{1}\right\}, H_{0}\right\}}{\left\{\left\{H_{0}, H_{1}\right\}, H_{1}\right\}}
$$

and we have:

Proposition 7: The singular extremals of the single-input case with $u_{2}=0$ are extremals of the bi-input case with the additional condition: $x_{1}=p_{x_{1}}=x_{2}=p_{x_{2}}=0$.

They are the extremals obtained numerically in the ideal contrast problem. Indeed in this case, the initial condition $q(0)$ corresponds to the North pole the Bloch ball $((0,0,1),(0,0,1))$ and we may choose to stay in the meridian plane given by $\left(x_{1}=x_{2}=0\right)$ imposing $u_{2}=0$ and the optimal solution is a sequence of bang and singular arcs associated with the single-input control system. The analysis of such a dynamics boils down to analyze the dynamics of the singular flow which is presented in the next section, using a specific example.

D. An example of the single-input case: algebraic classification in the multisaturation of the same species with $B_{1-}$ inhomogeneity

Next, we present a detailed analysis of the singular extremals, when one can restrict to the single-input case. The system is written $F_{0}+u_{1} F_{1}$ and $B_{1}$-inhomogeneity leads to a rescaling that is:

$$
\begin{aligned}
& F_{0}=\left(-\Gamma y_{1},-\gamma z_{1},-\Gamma y_{2},-\gamma z_{2}\right), \\
& F_{1}=\left(\left(-\left(z_{1}+1\right), y_{1}\right),(1-\varepsilon)\left(-\left(z_{2}+1\right), y_{2}\right)\right) .
\end{aligned}
$$

where for the sake of the computations the North pole of the Bloch ball $((0,1),(0,1))$ is taken as the origin of the coordinates. Moreover to simplify the computations we assume that the transfer time $t_{f}$ is not fixed and hence the 
singular extremals can be restricted to the level set $H_{0}=0$, thanks to the Maximum Principle. Using this constraint the singular control is given by the feedback:

$$
u_{1 s}(q)=-\frac{D^{\prime}(q)}{D(q)}
$$

with

$$
\begin{aligned}
D & =\operatorname{det}\left(F_{0}, F_{1},\left[F_{1}, F_{0}\right],\left[\left[F_{1}, F_{0}\right], F_{1}\right]\right), \\
D^{\prime} & =\operatorname{det}\left(F_{0}, F_{1},\left[F_{1}, F_{0}\right],\left[\left[F_{1}, F_{0}\right], F_{0}\right]\right)
\end{aligned}
$$

and plugging such $u_{1 s}$ leads to analyze: $F_{0}(q)-\frac{D^{\prime}(q)}{D(q)} F_{1}(q)$ and using a time parameterization the analysis is reduced to investigate the smooth vector field:

$$
X_{e}^{r}(q)=D(q) F_{0}(q)-D^{\prime}(q) F_{1}(q) .
$$

The crucial point is to compute the equilibrium points which is a complicated algebraic problem and the interest of our study is to use computer algebraic methods to handle this problem.

First of all, we have.

Lemma 3: 1) The quadric $D$ can be written as a sum of homogeneous forms $h_{i}$ of degree $i$

$$
D=h_{2}+h_{3}+h_{4}
$$

We set $D=(1-\varepsilon) \tilde{D}$.

2) The quadric $D^{\prime}$ reduces to a cubic form which is factorized into a linear and a quadratic (homogeneous) forms:

$$
\begin{aligned}
D^{\prime}=2 \gamma^{2}(\Gamma-\gamma)(2 \Gamma-\gamma)(1-\varepsilon) & \left(z_{1}-z_{2}\right) \\
& \left((\varepsilon-1) z_{1} y_{2}+z_{2} y_{1}\right) \triangleleft
\end{aligned}
$$

In particular we deduce:

Proposition 8: The quadric $D^{\prime}$ reduces to a cubic form which is factorized into a linear and a quadratic (homogeneous) forms.

a) Singular analysis: We assume $\gamma>0$ and $2 \Gamma>$ $3 \gamma$. It implies $\gamma \neq \Gamma$ and $\gamma \neq 2 \Gamma$. The main result is the following:

Theorem 1: Provided $\varepsilon \neq 1$ the equilibrium points of $X_{e}^{r}=D F_{0}-D^{\prime} F_{1}$ are all contained in $\left\{D=D^{\prime}=0\right\}$.

Proof: Obviously, every point of $\{D=0\} \cap\left\{D^{\prime}=0\right\}$ is a singularity of $X_{e}^{r}$.

Conversely, let us assume $\varepsilon \neq 1$. We first divide $X_{e}^{r}$ by $1-\varepsilon$. We still assume that $\Gamma \neq 0$. We consider the equations $\left\{\left(X_{e}^{r}\right)_{y_{1}}=0,\left(X_{e}^{r}\right)_{z_{1}}=0,\left(X_{e}^{r}\right)_{y_{2}}=0,\left(X_{e}^{r}\right)_{z_{2}}=\right.$ $0\}$ and remark that the last third are dividable by $\gamma$. By homogeneity, changing $\gamma$ into $\gamma \Gamma$, we get rid of $\Gamma$. So we may assume $\Gamma=1$. The resulting system is denoted $\Sigma_{r}$. We add the two polynomials $\left((\varepsilon-1) z_{1} y_{2}+z_{2} y_{1}\right) a_{1}-1$ and $\left(z_{1}-z_{2}\right) a_{2}-1$, and the polynomials $\gamma g-1,(\gamma-1) g_{1}-1$, $(\gamma-2) g_{2}-1$. We denote $\tilde{\Sigma}_{r}$ this new system, involving four new variables $g_{1}, g_{2}, a_{1}, a_{2}$. We compute a Gröbner basis with total degree with reverse lexicographic order on $\left(y_{1}, y_{2}, z_{1}, z_{2}, \varepsilon, g, g_{1}, g_{2}, a_{1}, a_{2}\right)$ and get $\{1\}$. Hence, provided $\gamma$ is different from $0,1,2$, there is no singular point of $X_{e}^{r}$ outside of $\{D=0\} \cap\left\{D^{\prime}=0\right\}$.
The remaining of the section is devoted to the singularity resolution. From the factorized form of $D^{\prime}$ (Proposition 8) we get:

Proposition 9: $\{D=0\} \cap\left\{D^{\prime}=0\right\}$ is an algebraic variety of algebraic dimension 2 whose components are located in the hyperplane $z_{1}=z_{2}$ and in the hypersurface $(\varepsilon-1) z_{1} y_{2}+z_{2} y_{1}=0$.

These components are studied in the following analysis, and explicitly expressed in Lemmas 4, 5, 6, 7 .

- Case A: components of $\{D=0\} \cap\left\{D^{\prime}=0\right\}$ in $z_{1}=z_{2}$. Under the constraint $z_{1}=z_{2}$, we have a factorization $\tilde{D}=p_{1} p_{2}$ with:

$$
p_{1}=2(\gamma-\Gamma) z_{1}+\gamma-2 \Gamma
$$

and:

$$
\begin{aligned}
p_{2}= & \left(2(\gamma-\Gamma)\left(\gamma+(\varepsilon-1)^{2} \Gamma\right) z_{1}+\Gamma(\varepsilon-1)^{2}\right. \\
& (\gamma-2 \Gamma)) y_{1}^{2}+\left(4(\gamma-\Gamma)(\gamma+\Gamma)(\varepsilon-1) z_{1}\right. \\
& +2 \Gamma(\varepsilon-1)(\gamma-2 \Gamma)) y_{2} y_{1}+(2(\gamma-\Gamma) \\
& \left.\left(\Gamma+(\varepsilon-1)^{2} \gamma\right) z_{1}+\Gamma(\gamma-2 \Gamma)\right) y_{2}^{2}
\end{aligned}
$$

The first polynomial has one root $z_{1}=z_{\gamma, \Gamma}$

$$
z_{\gamma, \Gamma}=\frac{1}{2} \frac{2 \Gamma-\gamma}{\gamma-\Gamma}
$$

which corresponds to the plane-solution $\left\{\left(y_{1}, z_{\gamma, \Gamma}, y_{2}, z_{\gamma, \Gamma}\right),\left(y_{1}, y_{2}\right) \in \mathbb{R}^{2}\right\}$.

We put:

$$
\begin{aligned}
d_{2}\left(y_{1}, y_{2}\right)= & \left(\gamma+(\varepsilon-1)^{2} \Gamma\right) y_{1}^{2}+2(\varepsilon-1) \\
& (\gamma+\Gamma) y_{2} y_{1}+\left(\Gamma+(\varepsilon-1)^{2} \gamma\right) y_{2}^{2} 1
\end{aligned}
$$

The discriminant of $d_{2}$ with respect to $y_{1}$ is $-4(\varepsilon-2)^{2} \gamma \Gamma \varepsilon^{2} y_{2}^{2}$ which is strictly negative provided $\varepsilon \neq 0$. So $d_{2}$ is non-zero outside $y_{1}=y_{2}=0$.

So, provided $y_{1}^{2}+y_{2}^{2} \neq 0, d_{2} \neq 0$, and $p_{2}=0$ is solved with respect to $z_{1}$. We get $z_{1}=r_{2}\left(y_{1}, y_{2}\right)$ with

$$
r_{2}\left(y_{1}, y_{2}\right)=\frac{\Gamma(2 \Gamma-\gamma)\left((\varepsilon-1) y_{1}+y_{2}\right)^{2}}{2(\gamma-\Gamma) d_{2}\left(y_{1}, y_{2}\right)}
$$

and $\left(y_{1}, r_{2}\left(y_{1}, y_{2}\right), y_{2}, r_{2}\left(y_{1}, y_{2}\right)\right) \quad$ (defined for $\left.\left(y_{1}, y_{2}\right) \neq(0,0)\right)$ vanishes both $D$ and $D^{\prime}$.

Finally, if $y_{1}=y_{2}=0$, we have the solution $(0, z, 0, z), \quad z \in \mathbb{R}$.

We summarize the case $z_{1}=z_{2}$ in:

Lemma 4: $\{D=0\} \cap\left\{D^{\prime}=0\right\} \cap\left\{z_{1}=z_{2}\right\}$ is the union of an affine plane $z_{1}=z_{2}=z_{\gamma, \Gamma}$, a rational surface $z_{1}=z_{2}=r_{2}\left(y_{1}, y_{2}\right)$ (defined for $\left(y_{1}, y_{2}\right) \neq$ $(0,0))$, and the line $\{(0, z, 0, z), z \in \mathbb{R}\}$.

- Case B: components of $\{D=0\} \cap\left\{D^{\prime}=0\right\}$ in $(\varepsilon-1) z_{1} y_{2}+z_{2} y_{1}=0$.

- Assume first that $y_{1}=0$ and $z_{1} \neq z_{2}$. We have $z_{1} y_{2}=0$.

* If $y_{1}=z_{1}=0$, then:

$$
\tilde{D}=(\gamma-2 \Gamma)\left(\Gamma(\gamma-2 \Gamma) y_{2}^{2}+\gamma^{2} z_{2}^{2}\right)
$$


Since $2 \Gamma>\gamma,\{\tilde{D}=0\} \cap\left\{y_{1}=z_{1}=0\right\}$ corresponds to two lines intersecting at $N$.

* If $y_{1}=y_{2}=0$, then let us put

$$
d_{1}\left(z_{1}\right)=2 \varepsilon(\varepsilon-2)(\gamma-\Gamma) z_{1}+2 \Gamma-\gamma .
$$

We have:

$$
\tilde{D}=\gamma^{2}\left(z_{2}-z-1\right)\left(d_{1}\left(z_{1}\right) z_{2}-(\varepsilon-1)^{2}(2 \Gamma-\gamma) z_{1}\right.
$$

Observe that the polynomial $d_{1}$ vanishes if and only if $z_{1}$ equals $\tilde{z}_{\gamma, \Gamma}$ with

$$
\tilde{z}_{\gamma, \Gamma}=\frac{1}{2} \frac{\gamma-2 \Gamma}{\varepsilon(\varepsilon-2)(\gamma-\Gamma)}
$$

and in this case, there is no solution such that $z_{2} \neq z_{1}$.

Provided $d_{1}\left(z_{1}\right) \neq 0$, one gets $z_{2}=r_{1}\left(z_{1}\right)$ :

$$
r_{1}\left(z_{1}\right)=\frac{(\varepsilon-1)^{2}(2 \Gamma-\gamma) z_{1}}{d_{1}\left(z_{1}\right)}
$$

which is a rational function of $z_{1}$. And the intersection with $\{D=0\} \cap\left\{D^{\prime}=0\right\}$ is the curve $\left\{\left(0, z_{1}, 0, r_{1}\left(z_{1}\right)\right) z_{1} \in \mathbb{R} \backslash\left\{\tilde{z}_{\gamma, \Gamma}\right\}\right\}$.

Lemma 5: $\{D=0\} \cap\left\{D^{\prime}=0\right\} \cap\left\{y_{1}=0\right\} \cap$ $\left\{\left(z_{1}-z_{2}\right) \neq 0\right\}$ is the union of two lines of $\left\{y_{1}=z_{1}=0\right\}$ intersecting at $\mathrm{N}$ and a rational curve $\left\{\left(0, z_{1}, 0, r_{1}\left(z_{1}\right)\right) z_{1} \in \mathbb{R} \backslash\left\{\tilde{z}_{\gamma, \Gamma}\right\}\right\}$.

- Let us assume $y_{1} \neq 0$.

We can eliminate $z_{2}$ using:

$$
z_{2}=\frac{z_{1} y_{2}(1-\varepsilon)}{y_{1}}
$$

and, substituting in $y_{1}^{2} \tilde{D}$ we get the factorization $y_{1}^{2} \tilde{D}=q_{1} q_{2}$, with:

$$
\begin{aligned}
q_{1}= & \Gamma(\varepsilon-1)(2 \Gamma-\gamma) y_{1}^{3}+\gamma^{2}(\varepsilon-1) z_{1}^{2} y_{1} \\
& +\gamma^{2}(\varepsilon-1)^{2} z_{1}^{2} y_{2}+(-\Gamma(\gamma-2 \Gamma) \\
& \left.+2 \Gamma \varepsilon(\varepsilon-2)(\gamma-\Gamma) z_{1}\right) y_{2} y_{1}^{2}
\end{aligned}
$$

and:

$$
\begin{array}{r}
q_{2}=(\varepsilon-1)(\gamma-2 \Gamma) y_{1}+(2 \varepsilon(2-\varepsilon) \\
\left.(\gamma-\Gamma) z_{1}+\gamma-2 \Gamma\right) y_{2}
\end{array}
$$

Provided $d_{1} \neq 0$ (that is $z_{1} \neq \tilde{z}_{\gamma, \Gamma}$ ), we solve $q_{2}=$ 0 with respect to $y_{2}$, and then we get the value of $\left(y_{2}, z_{2}\right)$ :

$$
\left(\frac{(\varepsilon-1)(\gamma-2 \Gamma) y_{1}}{d_{1}\left(z_{1}\right)}, \frac{(\varepsilon-1)^{2}(2 \Gamma-\gamma) z_{1}}{d_{1}\left(z_{1}\right)}\right)
$$

Lemma 6: $\{D=0\} \cap\left\{D^{\prime}=0\right\} \cap$ $\left\{\left(z_{1}-z_{2}\right) y_{1} d_{1}\left(z_{1}\right) \neq 0\right\}$ is a rational surface $\left(y_{2}=\rho_{2}\left(y_{1}, z_{1}\right), z_{2}=\rho_{1}\left(z_{1}\right) y_{1} \neq 0 z_{1} \neq \tilde{z}_{\gamma, \Gamma}\right) . \triangleleft$ We put $d_{3}$

$$
\begin{aligned}
d_{3}=\left(2 \Gamma \varepsilon(\varepsilon-2)(\gamma-\Gamma) z_{1}-\Gamma(\gamma-2 \Gamma)\right) y_{1}^{2} \\
+\gamma^{2}(\varepsilon-1)^{2} z_{1}^{2}
\end{aligned}
$$

Its discriminant with respect to $y_{1}$ is:

$$
-4\left(2 \Gamma-\gamma+2 \varepsilon(2-\varepsilon)(\Gamma-\gamma) z_{1}\right) \Gamma \gamma^{2} z_{1}^{2}(\varepsilon-1)^{2}
$$

and its sign changes when $z_{1}$ reaches $\tilde{z}_{\gamma, \Gamma}$. Provided $d_{3}\left(y_{1}, z_{1}\right) \neq 0$, we solve $q_{1}$ with respect to $y_{2}$, and then we get the value of $\left(y_{2}, z_{2}\right)$ :

$\left(\frac{\left(\Gamma(2 \Gamma-\gamma) y_{1}^{2}+\gamma^{2} z_{1}^{2}\right)(1-\varepsilon) y_{1}}{d_{3}\left(y_{1}, z_{1}\right)}, \frac{\left(\Gamma(2 \Gamma-\gamma) y_{1}^{2}+\gamma^{2} z_{1}^{2}\right)(\varepsilon-1)^{2} z_{1}}{d_{3}\left(y_{1}, z_{1}\right)}\right)$

Lemma 7: $\{D=0\} \cap\left\{D^{\prime}=0\right\} \cap$ $\left\{\left(z_{1}-z_{2}\right) y_{1} d_{3}\left(z_{1}\right) \neq 0\right\}$ is a rational surface with parameterization $\left(y_{2}=\rho_{3}\left(y_{1}, z_{1}\right), z_{2}=\rho_{4}\left(y_{1}, z_{1}\right)\right)$. $\triangleleft$

- Analysis of the behaviors of the solutions of $X_{e}^{r}$ near O.

We set $\tilde{z}_{i}=1+z_{i}$ and we have the following approximations:

$$
\begin{aligned}
& -\tilde{D}=h_{1}+h_{2}, \\
& h_{1}=\gamma^{2} \varepsilon(\varepsilon-2)(\gamma-2 \Gamma)\left(\tilde{z}_{1}-\tilde{z}_{2}\right) \\
& h_{2}=\Gamma(\varepsilon-1)^{2}(\gamma-2 \Gamma)^{2} y_{1}^{2}+\Gamma(\gamma-2 \Gamma)^{2} y_{2}^{2} \\
& \quad-\gamma^{2}(\gamma-2 \Gamma) \tilde{z}_{2}^{2}-\gamma^{2}(\varepsilon-1)^{2}(\gamma-2 \Gamma) \tilde{z}_{1}^{2} \\
& \quad+2 \Gamma(\gamma-2 \Gamma)^{2}(\varepsilon-1) y_{2} y_{1}+\gamma^{2}\left(\varepsilon^{2}+2\right. \\
& \quad-2 \varepsilon)(\gamma-2 \Gamma) \tilde{z}_{1} \tilde{z}_{2} \\
& -D^{\prime}=2 \gamma^{2}(\Gamma-\gamma)(2 \Gamma-\gamma)(1-\varepsilon)\left(\tilde{z}_{2}-\tilde{z}_{1}\right)[(-1+ \\
& \left.\left.\tilde{z}_{1}\right) y_{2}(\varepsilon-1)+\left(-1+\tilde{z}_{2}\right) y_{1}\right] .
\end{aligned}
$$

Conclusion: these computations allow to evaluate the equilibrium points and the behaviors of the solutions near such point, using linearization methods.

\section{REFERENCES}

[1] S. Conolly, D. Nishimura and A. Albert, Optimal control solutions to the magnetic resonance selective excitation problem, Medical Imaging, IEEE Transactions on, 5, no.2, 1986, pp.106-115.

[2] B. Bonnard and M. Chyba, Singular trajectories and their role in control theory, Springer-Verlag, Berlin, 2003.

[3] Bonnard, B., Claeys, M., Cots, O., Martinon, P.: Geometric and numerical methods in the contrast imaging problem in nuclear magnetic resonance. Acta Appl. Math. 135, no.1, 2015, pp.5-45

[4] B. Bonnard, O. Cots, S.J. Glaser, M. Lapert and D. Sugny, Geometric optimal control of the contrast imaging problem in nuclear magnetic resonance, IEEE Trans. Automat. Control, 57, no.8, 2012, pp.19571969.

[5] D. D'Alessandro, Introduction to quantum control and dynamics. Chapman \& Hall/CRC, Boca Raton, FL, 2008 pp. xiv+343.

[6] O. Cots, Contrôle optimal géométrique: méthodes homotopiques et applications, $\mathrm{PhD}$ thesis, Université de Bourgogne, 2012. http:// hampath.org

[7] S. Helgason, Differential geometry, Lie groups, and symmetric spaces, Academic Press, Inc., New York-London, 80, 1978.

[8] N. Khaneja, S.K. Glaser and R. Brockett Sub-Riemannian geometry and time optimal control of three spin systems: quantum gates and coherence transfer, Phys. Rev. A (3), 65, 2002, pp.032301.

[9] M. Lapert, Développement de nouvelles techniques de contrôle optimal en dynamique quantique : de la Résonance Magnétique Nucléaire à la physique moléculaire, Phd thesis, Laboratoire Interdisciplinaire Carnot de Bourgogne, Dijon (2011).

[10] J-S. Li and N. Khaneja, Ensemble control of Bloch equations, IEEE Trans. Automat. Control, 54, no.3, 2009, pp.528-536.

[11] M. Lapert, Y. Zhang, M.A. Janich, S.J. Glaser and D. Sugny Exploring the physical limits of saturation contrast in magnetic resonance imaging, Scientific Reports, 2, 2012.

[12] L.S. Pontryagin, V.G. Boltyanskii and R.V. Gamkrelidze, The Mathematical Theory of Optimal Processes, John Wiley and Sons, New York, 1962.

[13] T.E. Skinner, T. Reiss, B. Luy, N. Khaneja and S.J. Glaser, Application of optimal control theory to the design of broadband excitation pulses for high-resolution NMR, Journal of Magnetic Resonance, 163, no.1, 2003, pp.8-15. 